\title{
Neurocysticercosis - A Journey from Pre-independence to Modern India
}

\author{
Gagandeep Singh, Monika Singla \\ Department of Neurology, \\ Dayanand Medical College and Hospital, Ludhiana.
}

\begin{abstract}
Neurocysticercosis (NCC) is infestation of the human brain by the larva of worm, Taenia solium and is the most prevalent central nervous system (CNS) helminthiasis. The disease is widespread in tropical and subtropical regions of the world, including the Indian subcontinent, China, Sub-Saharan Africa, Central and South America and contributes substantially to the burden of epilepsy in these areas(1). CNS involvement is seen in $60-90 \%$ of systemic cysticercosis. About 2.5 million people worldwide are infected with $T$. solium, and antibodies to $T$. solium are seen in up to $25 \%$ of people in endemic areas (1-3). A higher prevalence of epilepsy and seizures in endemic countries is partly because of a high prevalence of cysticercosis in these regions. Seizures are thought to be caused by NCC in as many as 30\% of adult patients and in $51 \%$ of children in population based endemic regions (2). About $12 \%$ of admissions to neurological services in endemic regions are attributed to NCC and nearly half a million deaths occurring annually worldwide can be attributed directly or indirectly to NCC (Bern et al.). Punctate calcific foci on CT scan are a very common finding in asymptomatic people residing in endemic areas, found in 14-20\% of CT scans. Both seizures and positive cysticercus serology are associated with the detection of cysticerci on CT scans. Seroprevalence using a recently developed CDC- based enzyme-linked immunotransfer blot (EITB) assay is estimated at 8-12\% in Latin America and 4.9-24\% in Africa and South-East Asia. It is estimated that 20 million people harbour neurocysticercosis worldwide(1).
\end{abstract}

Key words : Neurocysticercosis (NCC), SCG, T. Solium, cysticerci.

Correspondence : Dr. Gagandeep Singh, Prof.\& Head, Department of Neurology, Dayanand Medical College and Hospital, Ludhiana - 141001 (Punjab). E.mail : gagandeep_si@yahoo.co.uk.

DR. BALDEV SINGH ORATION delivered during NAMSCON 2015 at the All-India Institute of Medical Sciences, Patna. 
Neurocysticercosis - A Journey from Pre-independence to Modern India 77

\section{INTRODUCTION}

Neurocysticercosis is the main cause of acquired (late-onset) epilepsy in many resource- poor countries(4). The clinical presentation, course and outcome of NCC depends on clinical subtype of NCC, which in turn is determined by several factors : the anatomical location of the cysticerci (parenchymal NCC, subarachnoid-racemose cysticerci, ventricular cysticerci, spinal cysticerci), number of cysticerci (single, few, disseminated) and evolutionary stage of the cysticerci(5). The latter, i.e., evolutionary stage is based on pathological classification of cysticercosis but has been adapted for use in clinical and imaging studies(6-7). The vesicular stage refers to the live or cystic or active stage of the cysticercus. The colloidal stage is also called transitional or degenerating stage. Likewise, the granular nodular stage represents the dying parasite. The fibro-calcified stage represents the dead or inactive parasite. The varied presentations of NCC are due to the several different combinations of location, number and stage of the cysticerci. For instance, multiple NCC, especially when the number of cysts is in 100 s presents with dementia and raised intracranial pressure (8-9). When multiple parenchymal cysticerci begin to degenerate, the clinical presentation is one of raised intracranial tension, seizures and focal neurological deficits. On the other hand, when only solitary or few cysticerci degenerate, the clinical presentation comprises of few seizures, that are usually easily controlled(10). These single or few cysticerci represent a relatively benign form of NCC. Other manifestations of NCC include communicating hydrocephalus, stroke-like presentation and cranial nerve deficits in the case of subarachnoid-racemose cysticercosis and obstructive hydrocephalus in the case of intraventricular NCC (11-12).

The lack of basic sanitation, contamination of available drinking water and crowded living conditions, often with animals living side by side- all of which render people at risk for developing cysticercosis in resource- poor regions of the world. In community-based studies in endemic regions, seizures are associated with pig-raising and positive cysticercus serology(13). Due to the epidemiology of the disease, it is particularly common in the slums.

Long-held concepts regarding the global burden of the disease, its morbidity and mortality and its prevalence in endemic areas have changed considerably following developments in the fields of epidemiology and neuroimaging. A major false assumption was the belief that NCC occurs only after eating raw or contaminated pork(14). It is now believed that any uncooked food may be contaminated with ova of $T$. solium. Contamination is known to occur when raw vegetables grown in land fertilised with human faeces are ingested, or when people harbouring tapeworms prepare food with hands containing the ova. This is borne out by the fact that areas with tradition of good sanitation do not report a high incidence of NCC, but regions with 
less developed sanitation systems may have as much as $10 \%$ prevalence of cysticercosis.

As computed tomography $(\mathrm{CT})$ and magnetic resonance (MR) imaging began to be routinely used in patients suffering from seizures, it became apparent that the prevalence of NCC was much greater than previously thought. Epilepsy was not known as a frequent symptom of NCC in the pre-CT era except for Dixon and Lipscomb's early study on British soldiers stationed in India. Definitive diagnosis of this condition could only be made post mortem until the use of imaging techniques coupled with serologic assays for the detection of parasite antigen in blood and cerebrospinal fluid (CSF). These methods enabled easy and accurate diagnosis of NCC in a large proportion of people with epilepsy, and led to recognition of this disease as a major public health problem worldwide.

Economic costs of NCC include costs of medical treatment, lost working days and loss due to livestock condemnation. In a study from India, the authors estimated the direct cost per patient with NCC treated with AEDs for the period until resolution of lesion to be Rs. 5916 or $41.4 \%$ of GNP per capita(15). Total costs including indirect costs were calculated to be Rs. 7273 per patient or $50.9 \%$ of GNP per capita. Based on these estimates, annual costs due to NCC in India have been pegged at Rs.104 million/year. In Mexico the corresponding figure stands at Rs. 3560 million and in Brazil at Rs.3400 million/year. Due to increasing migration into affluent countries from endemic areas, these nations also face a substantial disease burden: $10 \%$ of seizures in the SouthWestern USA are due to NCC and the annual loss due to NCC in the USA has been calculated to be US\$ 8.8 million.

The most important aspect of NCC prevention and control is proper sanitation and maintenance of personal hygiene(1617). Scientific pig rearing measures with proper feeding, antihelminthic treatment to pigs, and pig vaccination are being investigated as methods of eliminating $T$. solium from endemic communities. Inspection and proper cooking of pork is essential to prevent development of the carrier stage. Carriers may be treated with niclosamide or single dose praziquantel.

\section{Taenia solium: Life Cycle}

Man is the only definitive host for $T$. solium, the adult worm which inhabits the human jejunum and ileum. However, the developmental cycle of the parasite includes a larval stage in intermediate hosts, which are usually pigs but may by accident also be human beings (Fig. 1) $(5,18)$. Ova are liberated into the environment per anum in the form of eggcontaining segments (gravid proglottids) and shed from the body of the worm which may, by means of contaminated food or water, be ingested by another human host. The definitive host may also be infected through faecal-oral contamination as a result of poor hygiene and also by reverse peristalsis, which leads to the migration of ripe proglottids to the stomach. In either 
case, the eggs rupture to liberate hexacanth larvae (oncospheres) which burrow into the gastric mucosa and reach the portal venous system. From here, they are distributed across the blood stream to various organs. Once they reach small terminal vessels, the embryos establish and encyst to form the larval vesicles or cysticerci, reaching their definitive size in 2-3 months. They are then disseminated to various organs, preferentially to more vascular structures such as the ones like skeletal muscle, brain and eye. In the brain, parenchymal involvement is more common than extraparenchymal. Outside the nervous system, cysticercosis causes few symptoms as the parasitic cysts are destroyed by the host's immune response. Symptoms or signs usually result from parasites located in the eye or the nervous system. Pigs are infested by eggs or proglottids in the stools of a human tapeworm carrier.

The number of ingested ova, duration of exposure, and viability of ova influence the host response, clinical features and the course of the disease. Once lodged in host tissue the contents of the oncospheres liquefy leading to formation of the cysticercus.

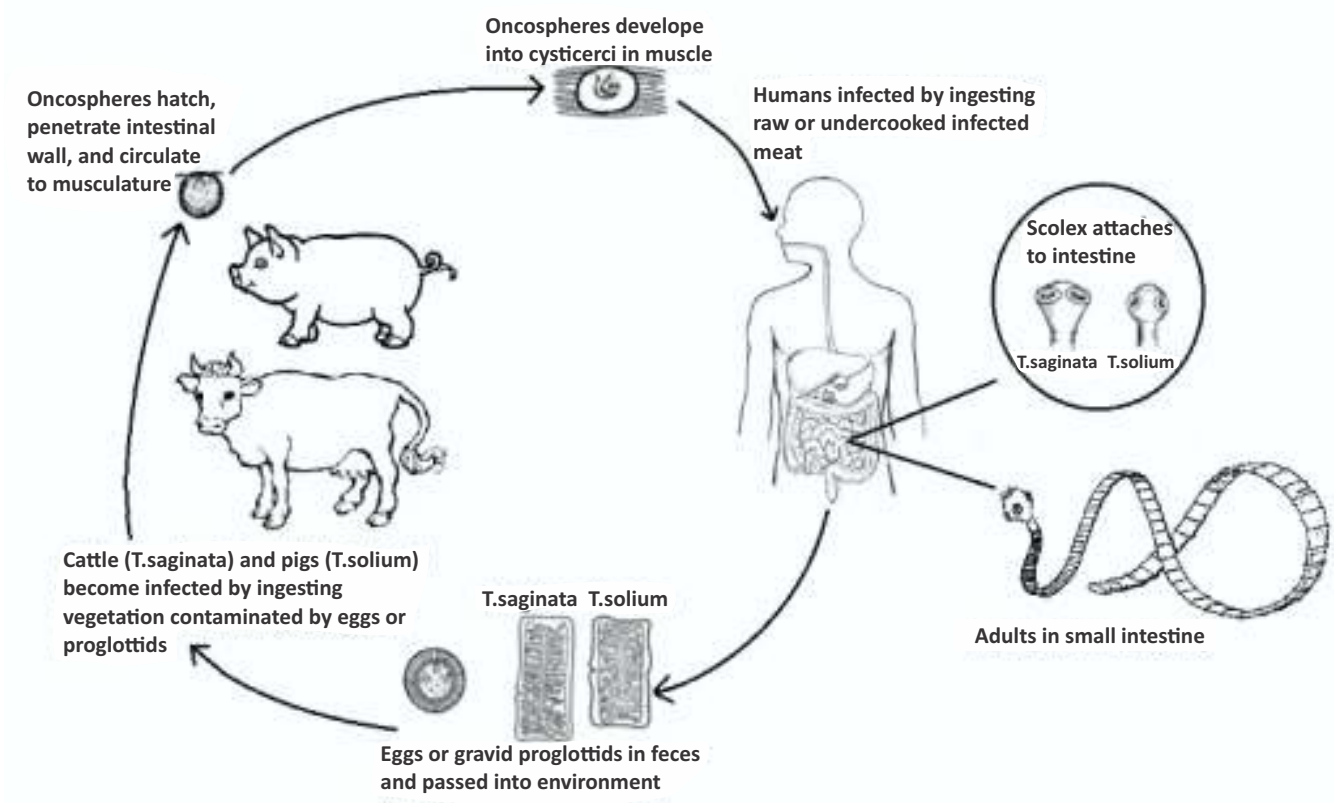

Fig. 1 : Figure depicting the life cycle of Taenia solium, going through various stages from egg to oncospheres to adult 


\section{Clinical Manifestations :}

Symptoms and signs of NCC infection of the human brain are several and varied(19-20). These may be delayed for years or may remain subclinical and depend on the host response, location number and size of the cyst/s(19). The mean duration between infection and presentation is 10.7 months (range 6-27 months) (11). Epilepsy is major problem in resource poor countries (with a prevalence of 5-10/1000 people), largely because of the high prevalence of NCC in these areas $(2,4)$. Following the advent of CT, Latin American studies have shown that over $30 \%$ of epilepsy, and over half of all late-onset seizures are related to $\operatorname{NCC}(2,4,21)$. Parenchymal NCC commonly presents with seizures (up to $90 \%)$ and headaches $(11,22)$. Seizures may be single, clustered or recurrent. They are either focal with or without secondary generalization or may be generalized at onset. Extraparenchymal cysts are usually not associated with seizures and these patients most commonly present with hydrocephalus, arachnoiditis, cranial nerve palsies and stroke-like episodes $(12,22,23)$.

In children too, seizures have been reported to be the commonest manifestation of NCC $(65.5 \%)$, followed by headache $(37.9 \%)$ and focal neurological deficits (24). Around 26$72 \%$ of children with a first seizure have a ring or disc lesion on $\mathrm{CT}$, which in most cases represents SCG (25).
Seizures may occur in any stage of NCC, but acute symptomatic seizures occur when a parenchymal cyst starts degenerating (symptomatic of the acute inflammatory response), and chronic epilepsy is perhaps due to gliosis around a chronic calcific lesion. Thus, seizures could occur due to several reasons in NCC. In resource poor countries, NCC is probably the commonest cause of acute symptomatic seizures; $92 \%$ of Indian patients with recurrent acute symptomatic seizures were due to NCC in one series (26). It has been proposed that seizures in the context of a degenerating cyst with oedema be considered acute symptomatic seizures, while those occurring in persons with calcified cysticerci are unprovoked (18).

\section{Classification of NCC:}

It is important to classify NCC as treatment and prognosis depends upon the classification. One way of classifying NCC is according to the pathological stages described by Escobar (6). Once a cysticercal cyst lodges in brain parenchyma, it undergoes spontaneous involution over varying periods. A parenchymal cyst typically goes through four morphological stages of involution: (a) vesicular; (b) colloidal; (c) granularnodular and (d) calcific. This entire process may take between few weeks and several years.

Patients may also be classified into those with active infection (with live cysticercus), transitional form (degenerating cyst) or inactive disease 
(dead parasite), although they frequently have multiple cysts simultaneously in varying stages of involution (7). Those patients with inactive infection may show calcification on CT, plain X-ray or MR: the former is thought to be most sensitive. They typically have a history of seizures in the past. However, the presence of such calcific foci is a known risk factor for seizure recurrence. Active parenchymal neurocysticercosis represents over $60 \%$ of cases in most series, presenting predominantly with seizures (22). On imaging, most patients show evidence of inflammation around the cysts in the form of oedema or enhancement of the cyst wall. Finally, depending upon the site of involvement, NCC may be classified into cranial (parenchymal, ventricular, subarachnoid - cisternal), spinal and mixed.

\section{Diagnosis of Neurocysticercosis :}

Accurate diagnosis of NCC is possible after interpretation of clinical data together with findings of neuroimaging studies and results of immunologic tests.

\section{Imaging Diagnosis:}

Computed tomography (CT) and magnetic resonance imaging (MRI) provide objective evidence about the topography of lesions, then evolutionary stage and degree of the host inflammatory response against cysticerci. CT is superior for detection of calcific lesions, whereas intraventricular, subarachnoid cysts and peri-cystic inflammation are better delineated by MRI. The intraventricular form presents with obstructive hydrocephalus, and only indirect signs like enlargement or deformation of ventricles or basal cisterns are seen on CT. MRI is the most accurate technique for imaging cysticercosis, providing the best evaluation of the degree of infestation, location and evolutionary stage of the parasite. MRI is more sensitive than CT scan for the diagnosis of neurocysticercosis, since it better recognizes the perilesional edema and the degenerative changes of the parasite, as well as small cysts or those located in the ventricles, brain stem, cerebellum, or the eyes, and the racemose vesicles at the level of the posterior fossae and basal cisterns. CT scan, however, is more sensitive for the detection of calcifications.

Most parenchymal lesions appear and show enhancement on contrast injection, usually surrounded by oedema. The vesicular stage, with a viable larva, appears as a smooth thin-walled cyst that is CSF-like on CT and MR images with no oedema or contrast enhancement. A mural nodule is often present that represents a viable larval scolex. When cyst degeneration begins (colloidal stage) and host inflammatory response ensues, pericystic oedema and cyst wall enhancement are present. Enhancement implies disruption of the blood-brain barrier. Cyst fluid is hyperintense compared to CSF on MR imaging during this stage. The colloidal stage has a thicker enhancing wall enclosing cyst fluid and prominent oedema. In the healing or granular nodular stage, nonenhanced CT 
scans show an iso-attenuated cyst with a hyperattenuated calcific scolex. Surrounding oedema is still present, and enhancement following contrast material administration persists. The residual cyst is isointense to the brain parenchyma on T1- weighted images and it is iso- to hypointense on T2-weighted images. Nodular or micro-ring enhancement is common at this stage, suggesting granuloma. Occasionally, a "target" appearance is seen with the calcified scolex in the centre of the mass. In the quiescent or residual stage, small calcified nodules without mass effect and usually without enhancement are seen.

Advantages of MRI over CT include: (a) capability of testing in different planes (axial, coronal, sagital); (b) it does not use iodine contrast and thus subjects are less prone to allergic reactions; (c) better sensitivity, except for calcifications; (d) it has various functional protocols (T1, T2, FLAIR, etc.) that allow for a better characterization of the parasite and of its evolutionary stage, and (e) no risk of radioactivity exposure.

\section{Serological Diagnosis :}

The enzyme-linked immunoelectrotransfer blot assay (EITB, Western Blot) provides specific confirmation of the diagnosis of NCC (27). In brief, this assay utilizes seven purified $T$. solium glycoprotein antigens (diagnostic bands GP50, GP42-39, GP24, GP21, GP18, GP14, and GP13) in an immunoblot format to detect infection-specific antibodies in serum or CSF samples.
Reactions to at least one band are considered positive. Specificity of this assay is $100 \%$, and its sensitivity in cases with viable parasites is estimated to be over $95 \%$ although it may be significantly lower in patients with a single cyst or a single degenerating parasite (28). Serologic tests are a valuable complement to neuroimaging in the evaluation of patients with suspected NCC, but they should not be used alone to exclude or confirm the diagnosis.

\section{Solitary Cysticercus Granuloma:}

A single enhancing CT lesion measuring less than $20 \mathrm{~mm}$ is a common finding upon CT of the brain of patients with seizures in India $(29,30)$. This lesion represents a solitary cysticercus granuloma (SCG) in the acute encephalitic phase. Epileptic seizures are by far the most common clinical manifestation of the SCG. While there is an over-abundance of reports of patients with SCG from India, these lesions have been reported from all over the world. In a hospital-based study from South India, this lesion accounted for $26 \%$ of etiological factors of symptomatic localization-related seizures (31) and 50\% of etiological factors for acute symptomatic seizures. The SCG is one of the commonest form of NCC. Its true incidence in comparison to other forms of NCC is not clear. In the hospital-based studies the reported frequency of solitary cyst (either granuloma alone, or SCG and solitary live cysts taken together) varied between $3.5 \%$ to $43 \%$ (32). In a study from Ecuador study, this lesion 
represented the single most common form of presentation of NCC, accounting for $23 \%$ of the cases (33).

\section{Historical Perspective:}

The solitary, small enhancing lesions, with surrounding edema were first reported as microtuberculomas or immature tuberculomas, by Bhargava and Tandon (34). There was no histological diagnosis at that time. Similar CT lesions were reported by some other Indian authors also. Wadia and colleagues also suggested similar lesions as microtuberculomas on the basis of a study on 39 patients with single small enhancing CT lesions (SSECTL), of whom ten patients had pulmonary tuberculosis (35). On this basis they advocated anti tubercular therapy for all patients with SSECTL. The diagnosis of microtuberculomas was first challenged by Sethi and colleagues (36), when they reported spontaneous resolution of lesions in patients with seizures, who did not take antitubercular treatment. These lesions were then described as Vanishing or Disappearing lesions in many reports (29, 37). Few authors considered these lesions as post-ictal as a result of breakdown of the blood brain barrier (29). Rajshekhar and colleagues performed stereotactic biopsy in patients with SSECTL and found evidence of cysticerci or merely parasitic material in several biopsy materials (38-41). The authors concluded that the majority of SSECTLs were solitary cysticercus granulomas (SCG). Subsequently, they evolved a set of diagnostic criteria for an initial diagnosis of SCG. These diagnostic criteria were found to have a sensitivity of $99.5 \%$, specificity of $98.9 \%$, positive predictive value of $99 \%$, and negative predictive value of $99.5 \%$ (42). Diagnostic criteria were further elucidated in a consensus paper (Table 1).

\section{Table 1.Clinical and radiologic features consistent with diagnose of SCG}

A. Clinical Feature that are supportive of diagnosis of SCG

Focal seizures or without secondary generalization

Note: Seizures may be new onset or of longer duration may be generalised at onset may occur in clusters ( 2 or more seizures over $2-3$ days) may be flowed by unilateral or diffuse headache lasting for a few hours to day/s or may be followed by transient and mild postictal neurological deficit.

B. Clinical features that make a diagnosis of SCG unlikely

Persistent and severe neurological deficit

Clinical evidence of intracranial hypertension

Evidence of neurologic disorder, other systemic disease (i.e systemic infection such as AIDS), that can account for imaging findings.

Age $<2$ years and $>60$ years

C. CT-features compatible with diagnosis of SCG (see Fig. 1)

Single small $(<20 \mathrm{~mm})$ well defined Contrast -enhancing (closed ring disc or nodular type) 
With or without surrounding edema Associated with minimal mass effect and no midline shift

D. MRI features compatible with diagnosis of SCG (see Fig. 1)

Single small $(<20 \mathrm{~mm}$ lesion with fluid contents)

T1 sequence intensity slightly greater than or isointense of CSF

T2 sequence hyperintense or isohypointense with central hyperintensity

Ring or nodular type enhancement after contrast

Scolex may or may not be visible as an eccentric nodule within the fluid cyst contents

( T1 isointense and T2 isohypointense)

Mild to moderate surrounding edema but no midline shift

\section{Abbreviation SCG-Solitary cysticercus granuloma}

These criteria were adapted from a review article, "A Diagnostic and therapeutic scheme for a Solitary Cysticercus Granuloma" (Neurology 75, December 14, 2010)(43).

\section{Treatment of Neurocysticercosis :}

\section{Antihelminthic Therapy:}

Although albendazole and praziquantel have been available since the 1980s and are relatively safe drugs with minimal toxicity and brief dosage schedules, even today there is considerable debate about their role in the treatment of NCC $(44,45)$. Anecdotal reports of the efficacy of the isoquinoline derivative praziquantel in NCC were available from the early 1980s, and an uncontrolled clinical trial in 1985 suggested its usefulness in patients with viable parenchymal cysticerci (46). Similarly, albendazole, a benzimidazole derivative has been used for human NCC since 1988 (47).

A head-to-head comparison between the two antihelminthic agents showed albendazole to be slightly superior, to have better penetration into the brain, and to cost less (47). The usual 30 -day duration of treatment $(15 \mathrm{mg} / \mathrm{kg} /$ day in 2 divided doses) is based on prior experience with human echinococcus, but serial MRI studies have demonstrated an equal benefit with an 8-day course (48) . Various studies have demonstrated equal efficacy with 15- and 17-day courses of albendazole, the outcome being measured by the change in cyst number and morphology on successive contrastenhanced CT scans (49). On cysticidal treatment including both praziquantel and albendazole, the problem is that patients may develop headache or vomiting during treatment due to the strong inflammatory reaction elicited in the brain due to larval degeneration induced by the antihelminthic agent. In one study conducted on patients with multiple cerebral cysticerci, adverse effects including nausea, vomiting and abdominal pain were seen in $38 \%$ of patients receiving albendazole. These were more severe with longer therapy. Headache was seen in $90 \%$ of patients at baseline and in $92 \%$ during 
therapy. $24 \%$ had seizures during treatment, all of which had prior history of seizures. Anti-inflammatory drugs and corticosteroids have been used widely to manage the antihelminthic drug-induced exacerbations. However, the routine use of steroids is not recommended, particularly as concomitant steroid administration reduces plasma levels of praziquantel. They may be given if the patient develops symptom or signs of intracranial hypertension during treatment.

As noted above, the effect of cysticidal therapy on the course and outcome of the disease has been a matter of considerable debate. The possible outcomes from the host parasite interaction in NCC include continued growth of the parasite, eventually producing a giant cyst, death of the cyst with resolution or calcification, and immune-mediated damage to the CNS parenchyma leading to neurologic symptoms and sequelae (50). Four main arguments have been put forth to support the view that cysticidal treatment is at best ineffective in preventing further seizures, and may even lead to clinical deterioration. First, the sudden destruction of parasites engendered by treatment may trigger an inflammatory reaction resulting in headache and seizures acutely, or an excess of gliosis in the long run leading to more seizures. Second, in a considerable fraction of patients with NCC, the disease is either clinically-silent or produced only mild symptoms (e.g., occasional seizures), which are easily managed. Third, in some patients the disease will be adequately eliminated either by host's response or spontaneous regression without the need for therapeutic intervention. Finally and fourth, the physical elimination of the parasite does not necessarily mean that the patient's neurologic dysfunction would improve. Against this has to be weighed the fact that therapy is safe, effective and convenient?

In 2004, the results of a randomised, placebo-controlled trial of albendazole in cystic parenchymal NCC were reported (51). This trial proved to be a benchmark in clinical trial research in NCC with its optimal study design, selection of outcome parameters and sound analysis. The study revealed that with treatment with albendazole, more patients demonstrated resolution of their lesions at six months post-treatment. At six months, $37 \%$ in the treated group were free of active lesions in comparison to $14 \%$ in placebo group. In addition, fewer patients in the treated group had partial seizures and significantly fewer patients had seizures with secondary generalization over a two-year period. Between 2 and 30 months of follow-up, $33 \%$ in the treated group in comparison to $48 \%$ in the placebo group had partial seizures (not significant). In the same period, $22 \%$ in the treated group had seizures with secondary generalisation in comparison to $37 \%$ in the placebo group ( $p=0.003)$. Thus, treatment with albendazole resulted in a reduction in the number of seizures and this effect was significant for seizures with secondary generalization. The authors of this study underscored the benefits of treatment with the argument 
that generalised seizures had a more devastating impact on the lives of the patients than partial seizures and hence, their reduction was a worthwhile outcome of treatment.

The trial demonstrated the benefits of treatment, these can best be construed to be modest and restricted to seizures with secondary generalisation (51). It may be argued that seizures, whether partial or generalised and the occurrence of either partial or generalised seizures precludes an individual from driving in most countries. The trial demonstrated that even after treatment with albendazole; about $2 / 3 \mathrm{rds}$ of the patients receiving albendazole continue to harbor active cysts, new drugs or different treatment regimens need to be explored for this majority of patients with NCC. Probably, studies are also required in order to understand why albendazole seems to work in some cases and why it does not in others. Appealing options include newer drugs (oxfendazole, for instance), combination of several anti-parasitic agents, either simultaneously or sequentially, and combination of antiparasitic agents with immunosuppressive agents.

Apart from these trials a number of other clinical trials have evaluated a variety of treatment regimens of albendazole and praziquantel. For instance, a seven-day course of albendazole has been compared with longer durations of treatment. A singleday treatment regimen with praziquantel was also evaluated. It was found to be of modest benefit in solitary cysticercus granuloma and not effective in multiple neurocysticercosis. Although, a large number of trials with antihelminthic treatment can be found in published literature, no firm conclusion can be derived from these early studies. Many of the studies had small number or subjects, were unrandomised or uncontrolled or used historical controls only. Other methodological problems have vitiated results in some studies, such as inclusion of cysts in various stages of development or of patients with chronic epilepsy and a lack of a clearly defined outcome.

In a meta-analysis, Otte and colleagues identified 10 randomised controlled trials of anti-parasitic treatment in patients with 1-2 enhancing lesions, involving 765 subjects, though there was considerable heterogeneity in the various studies regarding duration of antihelminthic (3-28 days), frequency of follow up and the radiological response (52). One single study compared single day praziquantel treatment as compared with placebo and demonstrated higher rate of resolution at 3 months. Though the placebo arms also demonstrated resolution of lesions at one year with symptomatic therapy alone but the rate of resolution was faster with albendazole. A single study reported 12 months follow up and no difference in seizure freedom. In the five studies, which reported follow up at 6 months, 180/199(90\%) were seizure free in the albendazole arm compared to placebo. Overall there was no significant difference in the frequency of calcifications at the end of follow up. 
Neurocysticercosis - A Journey from Pre-independence to Modern India 87

It appears that no standard treatment can be recommended for NCC. The parasitic disorder comprises several different presentations and treatment approaches depend on the clinical presentation, which in turn is determined by the location (parenchymal, intraventricular, subarachnoid-racemose, spinal), number of cysts and evolutionary stage of the cysticerci. Live (or cystic or vesicular) parenchymal cysticerci should be treated (Level 1 evidence). Evidence in favour of treatment of degenerating cysticerci (in the colloidal or granularnodular stage) is less convincing and probably needs further study. The calcified stage of cysticercosis does not require any antihelminthic treatment (Level 1 evidence). Treatment with antihelminthic drugs is contraindicated in disseminated cysticercosis, cysticercotic encephalitis and ocular cysticercosis. In the former case, treatment may lead to fatal intracranial hypertension, while in the latter case, treatment may lead to blindness.

In a recent trial, combination treatment (Albendazole and Praziquntel) was associated with significantly increased proportion of patients with complete resolution (64\% compared with $37 \%$ in standard albendazole group), which means a significantly improved rate of parasite clearance (53). The beneficial effects appeared to be restricted to people with multiple NCC. No difference in outcome was noted in people with single NCC.

\section{Corticosteroids :}

Corticosteroids have been used with a variety of indications in NCC. Benefits of their use are clearly discernable and often dramatic in some of the more severe forms of NCC such as cysticercotic encephalitis and subarachnoid-racemose cysticercosis. Furthermore, since the administration of albendazole often provokes seizures, focal neurological deficits and even intracranial hypertension in patients with parenchymal NCC due to the peri-cystic inflammation induced by cyst degeneration, it is routine clinical practice to co-administer corticosteroids with antihelminthic drugs. This practice of the co-administration of corticosteroids with albendazole is believed to reduce the incidence of adverse effects owing to their antiinflammatory actions. As a result, nearly all clinical trials of albendazole in NCC (SCG included) have incorporated corticosteroids in their intervention arm. The co-administration of the two agents does not allow us to dissect the benefits derived due to corticosteroids alone from those due to albendazole.

\section{Antiepileptic Drug Therapy :}

Seizures in NCC are managed in a manner similar to seizures of other etiologies. Carbamazepine is favored for treatment of partial seizures due to NCC; phenytoin is another drug that is often used (54). Drug rash with phenytoin has been reported to be more frequent in patients with SCG, and clobazam was used as a replacement with adequate 
seizure control (55). Another problem is that both carbamazepine and phenytoin induce the hepatic drug metabolizing enzymes that metabolize the antihelminthic agents, albendazole and praziquantel. This might decrease levels of the antihelminthic agents, thereby potentially compromising their cysticidal efficacy. Follow-up studies from Latin America of patients with multiple parenchymal NCC suggested a high frequency of breakthrough seizures and of seizure recurrence (nearly 50\%) following AED withdrawal after a period of seizure remission.

\section{Treatment of SSECTLS/SCGS :}

Single Small Enhancing CT Lesions (SSECTLs) are a common finding on computed tomography (CT) of young persons with new-onset seizures in India and also in several other parts of the world. It is currently believed that these lesions represent involuting cysticercus granuloma. In many ways, SSECTL / REL are a unique manifestation of neurocysticercosis (NCC). Although, these lesions have been noted in several geographical locations, they seem to be reported in large numbers from India. Thus, they may represent a geographically -dependant manifestation of NCC. They differ from lesions that have been studied in clinical trials described above in that they are encephalitic lesions as opposed to viable lesions. They also differ from lesions previously studied in clinical trials in being single as opposed to multiple. It has also been proposed that these lesions are biologically different from active and viable NCC by representing host inflammatory response to the embryonal stage of the parasite rather than to an established parasite.

\section{Role of Antihelminthics :}

Efficacy of antihelminthic drugs has been well established in live, vesicular parenchymal cysticercosis. The earliest RCT of albendazole in SCG could not find any difference in the frequency of resolution of the granuloma at 3 months among those who were treated with albendazole compared with those treated with nonspecific therapy. The previous meta-analysis of RCT's of albendazole in people with 1-2 parenchymal granuloma, reported in 2006, failed to demonstrate a better resolution rate with the use of albendazole (OR:1.18, 95\%, CI: 0.82 1.71, $\mathrm{P}=0.38$ ). Many more RCTs have undertaken after this meta-analysis. In the recent meta-analysis of $10 \mathrm{RCTs}$ by Otte et al., involving 765 subjects with SCG, of antihelminthic treatment (52, 56-65), some subjects had 2 rather than a single enhancing lesion in one study. This metaanalysis was different from the previous meta-analysis as it analysed pooled data according to the time of outcome assessment in the component RCT's (i.e $3,6 \& 12$ months). A significantly higher rate of granuloma resolution was observed with antihelminthic treatment from 3 and 6 months (OR: 2.09 ; 95\% CI: 1.41-3.09; p $\left.=0.0003 ; \mathrm{I}^{2}=31 \%\right)$. When trials with repeated-measures design were excluded from analysis, the ORs for granuloma resolution remained increased in favour of antihelminthic treatment both at 3 months (OR: $2.25 ; 95 \%$ CI: $1.43-3.52 ; \mathrm{p}=$ 
$0.0004 ; \mathrm{I}^{2}=12 \%$ (data removed from 6 months) and 6 months (OR: $2.10 ; 95 \% \mathrm{CI}$ : $\left.1.28-3.47 ; p=0.004 ; I^{2}=41 \%\right)$. As the chances of resolution clearly increase with longer follow up, therefore compared with the previous meta-analysis, this recent analysis established the benefit of antihelminthic treatment with albendazole both at 3 and 6 months and in overall pooled analysis.

\section{Seizure recurrence on antihelminthic} treatment:

The proportion of subjects with seizure recurrence on antiepileptic drugs (AEDs) after therapeutic intervention with antihelminthic drugs was reported for 3-month follow-up in 2 RCTs $(57,61)$, 6-month follow-up in 5 RCTs $(58,60,61$, $64,65)$ and 12 -month follow-up in 1 RCT (59). The proportion of subjects who remained seizure free was significantly higher in the antihelminthic treated group compared with controls for 3 months follow-up (non-event OR [i.e., 1/OR for seizure recurrence]: $4.05 ; 95 \% \mathrm{CI}$ : $1.76-9.33 ; p=0.001 ; \mathrm{I}^{2}=0 \%$ ), but not for 6 months (non-event OR: 1.79; 95\% CI: $0.95-3.38 ; \mathrm{p}=0.45 ; \mathrm{I}^{2}=0 \%$ ) or 12 months (non-event OR: 0.64; 95\% CI: 0.19-2.17; $\mathrm{p}=0.47$; heterogeneity not applicable). Meta-analysis of the pooled data from trials revealed significantly higher seizure-freedom rates at 3 and 6 months in the pooled antihelminthic-treated group compared with the pooled controls (nonevent OR: $2.45 ; 95 \%$ CI: $1.49-4.03 ; \mathrm{p}=$ 0.0004 ), therefore in assessing seizures freedom also, the antihelminthic treatment was found to be beneficial.

\section{Residual calcification with antihelminthic treatment:}

Though the residual calcification is also associated with occurrence of long term seizures, these was no evidence of ether increased or decreased risk of residual calcification with antihelminthic treatment.

\section{Conclusion :}

In conclusion, the use of albendazole (with or without corticosteroids) is recommended in the treatment of SCGs because it offers increased possibility of seizure freedom in patients with SCGs and improve rates of granuloma resolution.

\section{Role of corticosteroids :}

The notion that corticosteroids alone could alter the clinical course and outcome of SCG arose out of anecdotal observations of their use in providing symptomatic benefit in patients with SCG. Symptoms such as headaches and seizure exacerbations have been found to resolve with the administration of corticosteroids. The routine use of oral corticosteroids alone (without specific antihelminthic treatment) in the management of SCG is advocated by certain authors. Otte et al., in their recent meta-analysis, included five RCTs, involving 457 people with SCG, of corticosteroid treatment (66-70).

In various separate clinical trials, the administration of either prednisolone (orally, $1 \mathrm{mg} / \mathrm{kg} / \mathrm{d}$ for 10 days) alone (with 
antiepileptic drugs) or intravenous methyl prednisolone (one gram/d for five days) was associated with clinical and/or radiological benefits over 6-9 monthperiods (66-70). There were some discrepancies in the outcomes of these trials, but these appeared mainly due to the small size of the individual trials and when data from these trials were pooled beneficial effects were quite apparent. The administration of corticosteroids through either route resulted in significantly better rates of complete resolution of the SCG at six months and significantly fewer patients with seizure recurrence over a 6-9 months period. All these trials, however were located at one centre only and thus essentially represent a single-centre experience. Another shortcoming was that seizure recurrence was not clearly defined in these trials. Seizure recurrences in SCG are the result of brain parenchymal inflammation that follows cyst degeneration. The latter has been found to be a phasic rather than a continuous process. In keeping with this hypothesis, are the observations that seizures are often clustered in SCG and are related chronologically to the development of oedema surrounding, and of contrast enhancement of cysticercus lesions in the brain parenchyma, when followed over long periods of time. Hence the administration of corticosteroids may reduce the incidence of breakthrough seizures owing to their anti-inflammatory effects.

\section{Granuloma resolution with corticosteroid treatment :}

Five RCTs measured the effect of treatment with a short course of corticosteroids alone (standard AED treatment) on granuloma resolution (6670). Three RCTs reported rates of granuloma resolution at 3 months $(69,70)$ and 3 at 6 months $(66,68,70)$ of follow-up time. One RCT used a repeated-measures design with different modalities, CT at 3 months and MRI at 6 months (70). A random-effects analysis (performed because of significant heterogeneity; $\mathrm{I}^{2}=$ $74 \%$ ) did not reveal any significant difference in the rates of granuloma resolution over 3 and 6 months as well as in the overall combined analysis (OR: 1.82; 95\% CI: $0.88-3.77$; $\mathrm{p}=0.11$ ). This non-significant pooled effect remained using a sensitivity analysis when the repeated-measures data were excluded from the 3-month analysis (OR: 1.99; 95\% CI: $0.79-5.04 ; \mathrm{p}=0.15)$ and the 6month analysis (OR: 1.98; 95\% CI: $0.78-5.00 ; \mathrm{p}=0.15)$.

\section{Residual calcification with corticosteroid treatment :}

Three RCTs of corticosteroid in SCG reported rates of residual calcification on follow-up imaging studies $(67,68,70)$. The proportions of subjects with residual calcification on follow-up imaging were similar in the 2 pooled groups using fixed-effects analysis (OR: 1.18; 95\% CI: $0.54-2.56 ; \mathrm{p}=0.68 ; \mathrm{I}^{2}=$ $0 \%$ ). Subgroup heterogeneity was not significant $\left(p=0.25 ; I^{2}=25.7 \%\right)$. 


\section{Conclusion :}

In conclusion, the pooled evidence does not confirm a beneficial effect of the administration of corticosteroids alone in the treatment of SCGs.

\section{Corticosteroids alone versus Albendazole in conjunction with Corticosteroids :}

Two open-labelled clinical trials compared the administration of albendazole with prednisolone with the administration of prednisolone alone (with antiepileptic drugs) for short periods of time upon presentation(61). In both trials, the rates of cyst resolution (defined as complete disappearance of the SCG) at six months were similar in the groups administered corticosteroids alone and corticosteroids and albendazole. The two trials were inconsistent in the differential effects of the two interventions on the number of patients with seizure recurrence on follow-up. One reported increased rates of seizures, whilst the other reported decreased rates of seizures with the administration of corticosteroids alone. These differences could be due to differences in trial methods and the small number of patients recruited in either trial. Nonetheless, these trials likewise underscore the need for a large clinical trial comparing the effects of corticosteroids alone versus the administration of corticosteroids with antihelminthic drugs. A trial of such design with adequate number of subjects alone could dissect out the beneficial effects of corticosteroids from that of antihelminthic drugs.
It is not clear whether the reduced risk of seizures was owing to a symptomatic anti-inflammatory effect of the corticosteroids or a long-term effect by altering the natural history of the granuloma. It is likely that corticosteroids had a long term modifying effect of the disease course in as much as CT performed at one month revealed significantly greater chances of complete resolution. It is known that the occurrence of seizures is related to the persistence of a lesion in the case of parenchymal NCC. Hence, the reduced incidence of seizures in the treated group might have been due to an earlier resolution of the granuloma with corticosteroid administration.

Whilst prescribing corticosteroid to patients with NCC, one should be guided by the basic principle of their administration, i.e., "to achieve and maintain a satisfactory effect while using the lowest possible dose for the shortest time". One also needs to be aware of the drug interactions involved in such a therapeutic undertaking. Dexamethasone decreases praziquantel levels, potentially compromising its efficacy and thereby necessitating higher doses of the latter. On the other hand, dexamethasone decreases plasma albendazole-sulfoxide levels. Finally, antiepileptic drugs, including phenytoin and phenobarbitone induce the metabolism of corticosteroids. Consideration should also be made of the side-effects of corticosteroids; these include behavioural side effects and central serous retinopathy over the short term and diabetes, hypertension and bone loss over the long term. 


\section{Antiepileptic Drugs :}

At least three short-term (up to one year follow-up), randomised studies have demonstrated that seizure recurrence rates are not much different between patients with solitary cysticercus granuloma administered short-duration (six monthsone year) in comparison to longer duration (up to two years) of AED treatment (7173). Longer duration (up to five years) seizure remission rates with short-term AED treatment have also been studied; these are about $85 \%$, suggesting that seizure prognosis is essentially good (74). These follow-up studies have uniformly demonstrated that the single most important factor that determines seizure recurrence is the presence of a persisting lesion, whether a persisting involuting granuloma or a calcified residue. The likelihood of seizure recurrence in the event of complete resolution of the cysticercus granuloma is very low.

It seems reasonable to administer AEDs to patients with solitary cysticercus granuloma till such time that the lesion persists upon imaging studies. Once the lesion resolves completely, the AEDs can be safely withdrawn. In the case of residual calcification, longer duration of AED treatment may be required, but the duration of such treatment is not clear. Seizures in the setting of a calcified residue may occur owing to the intermittent release of antigenic material from within the calcified, dormant granuloma (75). This antigenic release causes acute symptomatic seizures due to cerebral irritation and manifests on imaging studies as contrast enhancement of the lesion and surrounding oedema. This may occur several years after the initial resolution of the granuloma. What proportion of calcific residues of cysticercus granulomas demonstrate this phenomena and for how long, remains to be settled by very long term follow-up studies.

The presence of perilesional gliosis on magnetisation transfer-MRI in the aftermath of a solitary cysticercus granuloma has been shown to be associated with seizure recurrence in one study (76). Residual gliosis demonstrated on either, magnetisation transfer imaging or fluid attenuated inversion recovery sequences may thus be a marker for seizure recurrences. The authors suggested that the demonstration of gliosis should dictate long-term AED treatment. Again longer duration, randomised studies of AEDs will be able to determine the need for, and duration of, AED therapy in such cases.

There is paucity of literature with regard to the choice of AEDs in NCC. Although, the range of options a propos, the choice of AEDs in epilepsy in general, also applies to NCC, it is desirable to administer an AED with a rapid onset of action. This is so because, seizures due to NCC are provoked by ongoing brain inflammation and are often clustered. Hence, there is a high risk of seizure recurrence in the immediate aftermath of a seizure. Thus, phenytoin-sodium in oral or parenteral loading doses is the preferred agent. However, one needs to be aware of 
the adverse effects to this agent. In particular, we and other authors have reported a high incidence of anticonvulsant hypersensitivity syndrome in individuals with solitary cysticercus granuloma who were administered Phenytoin (55). A recent randomised, open-labelled trial demonstrated equivalent efficacy and lesser incidence of drug rash with clobazam treatment in comparison to phenytoin in patients with solitary cysticercus granuloma (77). However, the cost of treatment with clobazam is higher; this needs to be kept in mind, especially so whilst treating patients with NCC, a disease that largely affects the resource-poor sections.

\section{Conclusion :}

In conclusion, the risk of seizure recurrence remains high if there is persisting granuloma or resolves leaving behind a calcific residue. In this case, the longer duration of AED should be considered, though the exact duration of therapy is unclear. Any AED might be used for this recommended period of treatment in individuals with SCG. However a newer, non-enzyme-inducing AED might be considered for the period of time that antihelminthic treatment is administered.

\section{REFERENCES :}

1. Bern C, Garcia HH, Evans C, et al. (1999). Magnitude of the disease burden from neurocysticercosis in a developing country. Clin Infect Dis 29(5): 1203-1209.
2. Montano SM, Villaran MV, Ylquimiche L, et al. (2005). Neurocysticercosis: association between seizures, serology, and brain CT in rural Peru. Neurology 65(2):229-233.

3. Del Brutto $\mathrm{OH}$, Santibanez R, Noboa CA, Aguirre R, Diaz E, Alarcon TA (1992). Epilepsy due to neurocysticercosis: analysis of 203 patients. Neurology 42(2):389-392.

4. Medina MT, Rosas E, RubioDonnadieu F, Sotelo J (1990). Neurocysticercosis as the main cause of late-onset epilepsy in Mexico. Arch Intern Med 150(2):325-327.

5. Garcia HH, Del Brutto $\mathrm{OH}$, Cysticercosis Working Group in Peru (2005). Neurocysticercosis: updated concepts about an old disease. Lancet Neurol 4(10):653661.

6. Escobar A, Aruffo C, Cruz-Sanchez F, Cervos-Navarro J (1985). Neuropathologic findings in neurocysticercosis. Arch Neurobiol (Madr.) 48(3): 151-156.

7. Carpio A, Placencia M, Santillan F, Escobar A (1994). A proposal for classification of neurocysticercosis. Canadian J Neurologi Sci 21(1):4347. 
8. Garcia HH, Del Brutto OH (1999). Heavy nonencephalitic cerebral cysticercosis in tapeworm carriers. The Cysticercosis Working Group in Peru. Neurology 53(7):15821584.

9. Wadia N, Desai S, Bhatt M (1988). Disseminated cysticercosis. New observations, including CT scan findings and experience with treatment by praziquantel. Brain 111 (Pt 3):597-614.

10. Rajashekhar V (2003). Solitary cerebral cysticercus granuloma. Epilepsia 44 Suppl 1: 25-28.

11. McCormick GF, Zee CS, Heiden J (1982). Cysticercosis cerebri. Review of 127 cases. Arch Neurol 39(9):534-539.

12. Grisolia JS, Wiederholt WC (1982). CNS cysticercosis. Arch Neurol 39(9):540-544.

13. Garcia HH, Gilman RH, Gonzalez AE, Pacheco R, Verastegui M, Tsang VC (1999). Human and porcine Taenia solium infection in a village in the highlands of Cusco, Peru. The Cysticercosis Working Group in Peru. Acta Trop 73(1):3136.

14. Garcia HH, Gonzalez AE, Del Brutto OH, et al. (2007). Strategies for the elimination of taeniasis/ cysticercosis. J Neurol Sci 262(12):153-157.
15. Murthy JM, Rajshekar G (2007). Economic evaluation of seizures associated with solitary cysticercus granuloma. Neurology India 55(1):42-45.

16. Pawlowski Z, Allan J, Sarti E (2005). Control of Taenia solium taeniasis/cysticercosis: from research towards implementation. Int J Parasitol 35(11-12):12211232.

17. Schantz PM (2006). Progress in diagnosis, treatment and elimination of echinococcosis and cysticercosis. Parasitol Intl $\mathbf{5 5}$ Suppl:S7-S13.

18. Garcia HH, Del Brutto OH (2000). Taenia solium cysticercosis. Infect Dis Clin North Am 14(1):97-119, ix.

19. Garcia HH, Del Brutto OH, Nash TE, White AC Jr, Tsang VC, Gilman RH (2005). New concepts in the diagnosis and management of neurocysticercosis (Taenia solium). Am JTrop Med Hyg 72(1):3-9.

20. Carpio A (2002). Neurocysticercosis: an update. Lancet Infect Dis 2(12):751-762.

21. Rajshekhar V, Raghava MV, Prabhakaran V, Oommen A, Muliyil J (2006). Active epilepsy as an index of burden of neurocysticercosis in Vellore district, India. Neurology 67(12):21352139. 
Neurocysticercosis - A Journey from Pre-independence to Modern India 95

22. Sotelo J, Guerrero V, Rubio F (1985). Neurocysticercosis: a new classification based on active and inactive forms. A study of 753 cases. Arch Intern Med 145(3):442445.

23. Wiederholt WC, Grisolia JS (1982). Cysticercosis : An old scourge revisited. Arch Neurol 39(9):533.

24. Mitchell WG (1997). Pediatric neurocysticercosis in North America. Eur Neurol 37(2):126129.

25. Mitchell WG, Crawford TO (1988). Intraparenchymal cerebral cysticercosis in children: diagnosis and treatment. Pediatrics 82(1):7682.

26. Singh G, Singh P, Singh I, Rani A, Kaushal S, Avasthi G (2006). Epidemiologic classification of seizures associated with neurocysticercosis: observation from a sample of seizure disorders in neurologic care in India. Acta Neurol Scand 113(4):233-240.

27. Tsang VC, Brand JA, Boyer AE (1989). An enzyme-linked immunoelectrotransfer blot assay and glycoprotein antigens for diagnosing human cysticercosis (Taenia solium). J Infect Dis 159(1):50-59.
28. Singh G, Kaushal V, Ram S, Kaushal RK, Dhanuka AK, Khurana S (1999). Cysticercus immunoblot assay in patients with single, small enhancing lesions and multilesional neurocysticercosis. $J$ Assoc Physicians India 47(5):476479.

29. Ahuja GK, Behari M, Prasad K, Goulatia RK, Jailkhani BL (1989). Disappearing CT lesions in epilepsy: Is tuberclosis or cysticercosis the cause ? J Neurol Neurosurg Psychiatry 52(7) : 915916.

30. Garg RK (2002). Single enhancing computerized tomography detected lesion in immunocompetent patients. Neurosurg Focus 12(6):e4.

31. Murthy JM, Yangala R (1998). Etiological spectrum of symptomatic localization related epilepsies: A study from South India.J Neurol Sc 158(1):65-70.

32. Garg RK, Agrawal A, Verma M (1991). CT spectrum of neurocysticercosis. J Assoc Physicians India 39(9):726-727.

33. Del Brutto OH (1995). Single parenchymal brain cysticercus in the acute encephalitic phase: definition of a distinct form of neurocysticercosis with a benign prognosis. J Neurol Neurosurg Psychiatry 58(2):247-249. 
34. Bhargava S, Tandon PN (1980). Intracranial tuberculomas: a CT study. Brit J Radiol 53(634):935945.

35. Wadia RS, Makhale CN, Kelkar AV, Grant KB (1987). Focal epilepsy in India with special reference to lesions showing ring or disc-like enhancement on contrast computed tomography. J Neurol Neurosurg Psychiatry 50(10):1298-1301.

36. Sethi PK, Kumar BR, Madan VS, Mohan V (1985). Appearing and disappearing CT scan abnormalities and seizures. $J$ Neurol Neurosurg Psychiatry 48(9):866-869.

37. Chopra JS, Sawhney IM, Suresh N, Prabhakar S, Dhand UK, Suri S (1992). Vanishing CT lesions in epilepsy. JNeurol Sci 107(1):40-49.

38. Chandy MJ, Rajshekhar V, Prakash $\mathrm{S}$, et al. (1989). Cysticercosis causing single, small CT lesions in Indian patients with seizures. Lancet 1(8634):390-391.

39. Rajshekhar V, Chacko G, Haran RP, Chandy MJ, Chandi SM (1995). Clinicoradiological and pathological correlations in patients with solitary cysticercus granuloma and epilepsy: focus on presence of the parasite and oedema formation. $J$ Neurol Neurosurg Psychiatry 59(3):284-286.
40. Rajshekhar V, Chandy MJ (1996). Comparative study of CT and MRI in patients with seizures and a solitary cerebral cysticercus granuloma. Neuroradiology 38(6): 542-546.

41. Rajshekhar V (1991). Etiology and management of single small CT lesions in patients with seizures: understanding a controversy. Acta Neurol Scand 84(6):465-470.

42. Rajshekhar V, Chandy MJ (1997). Validation of diagnostic criteria for solitary cerebral cysticercus granuloma in patients presenting with seizures. Acta Neurol Scand 96(2):76-81.

43. Singh G, Rajshekhar V, Murthy JM, et al. (2010). A diagnostic and therapeutic scheme for a solitary cysticercus granuloma. Neurology 75(24):2236-2245.

44. Robles C (1982). Mortality in 100 patients with neurocysticercosis treated with praziquantel. Salud publica a Mex 24(6):629-632.

45. Robles C, Sedano AM, VargasTentori N, Galindo-Virgen S (1987). Long-term results of praziquantel therapy in neurocysticercosis. J Neurosurgery 66(3):359-363. 
46. Sotelo J, Torres B, RubioDonnadieu F, Escobedo F, Rodriguez-Carbajal J (1985). Praziquantel in the treatment of neurocysticercosis: long-term follow-up. Neurology 35(5):752755 .

47. Sotelo J, Escobedo F, Penagos P (1988). Albendazole vs praziquantel for therapy for neurocysticercosis. A controlled trial. Arch Neurol 45(5):532-534.

48. Sotelo J, Penagos P, Escobedo F, Del Brutto OH (1988). Short course of albendazole therapy for neurocysticercosis. Arch Neurol 45(10):1130-1133.

49. Sotelo J, Del Brutto OH (1987). Therapy of neurocysticercosis. Child's nervous system : ChNS : official journal of the International Society for Pediatric Neurosurgery. 3(4):208-211.

50. Garcia HH, Gonzalez AE, Evans CA, Gilman RH, Cysticercosis Working Group in Peru (2003). Taenia solium cysticercosis. Lancet 362(9383):547-556.

51. Garcia HH, Pretell EJ, Gilman RH, et al. (2004). A trial of antiparasitic treatment to reduce the rate of seizures due to cerebral cysticercosis. NEJM 350(3):249258.
52. Otte WM, Singla M, Sander JW, Singh G (2013). Drug therapy for Solitary Cysticercus Granuloma. Neurology 80: 152-162.

53. Singh G, White AC Jr. (2014). Determining better treatments for neurocysticercosis. Lancet Infect Dis 14: 658-659.

54. Nash TE, Singh G, White AC, et al. (2006). Treatment of neurocysticercosis: current status and future research needs. Neurology 67(7):1120-1127.

55. Singh G, Kaushal S, Gupta M, Chander Chopra S (2004). Cutaneous reactions in patients with solitary cysticercus granuloma on phenytoin sodium. J Neurol Neurosurg Psychiatry 75(2):331333.

56. Alarcon F (2001). Short course of albendazole therapy for neurocysticercosis: a prospective randomized trial comparing three days, eight days, and a control group without albendazole. Rev Ecuat Neurol 10:1-2.

57. Baranwal AK, Singhi PD, Khandelwal N, Singhi SC (1998). Albendazole therapy in children with focal seizures and single small enhancing computerized tomographic lesions: a randomized, placebo-controlled, double blind trial. Pediatr Infect Dis J 17(8):696700 . 
58. Chaurasia RN, Garg RK, Agarwall A, et al. (2010). Three day albendazole therapy in patients with a solitary cysticercus granuloma: a randomized double blind placebo controlled study. Southeast Asian J Trop Med Public Health 41(3):517525.

59. de Souza A, Nalini A, Kovoor JM, Yeshraj G, Siddalingaiah HS, Thennarasu K (2011). Perilesional gliosis around solitary cerebral parenchymal cysticerci and longterm seizure outcome: a prospective study using serial magnetization transfer imaging. Epilepsia 52(10) :1918-1927.

60. Gogia S, Talukdar B, Choudhury V, Arora BS (2003). Neurocysticercosis in children: clinical findings and response to albendazole therapy in a randomized, double-blind, placebo-controlled trial in newly diagnosed cases. Trans $R$ Soc Trop Med Hyg 97(4):416-421.

61. Kalra V, Dua T, Kumar V (2003). Efficacy of albendazole and shortcourse dexamethasone treatment in children with 1 or 2 ring-enhancing lesions of neurocysticercosis: a randomized controlled trial. $J$ Pediatr 143(1):111-114.

62. Padma MV, Behari M, Misra NK, Ahuja GK (1994). Albendazole in single CT ring lesions in epilepsy. Neurology 44(7):1344-1346.
63. Pretell EJ, Garcia HH, Custodio N, et al. (2000). Short regimen of praziquantel in the treatment of single brain enhancing lesions. Clin Neurol Neurosurg 102(4):215-218.

64. Singhi P, Jain V, Khandelwal N (2004). Corticosteroids versus albendazole for treatment of single small enhancing computed tomographic lesions in children with neurocysticercosis. J Child Neurol 19(5):323-327.

65. Thussu A, Chattopadhyay A, Sawhney IM, Khandelwal N (2008). Albendazole therapy for single small enhancing $\mathrm{CT}$ lesions (SSECTL) in the brain in epilepsy. $J$ Neurol Neurosurg Psychiatry 79(3):272-275.

66. Garg RK, Potluri N, Kar AM, et al. (2006). Short course of prednisolone in patients with solitary cysticercus granuloma: a double blind placebo controlled study. JInfect 53(1):65-69.

67. Kishore D, Misra S (2007). Short course of oral prednisolone on disappearance of lesion and seizure recurrence in patients of solitary cysticercal granuloma with single small enhancing CT lesion: an open label randomized prospective study. JAPI 55:419-424. 
68. Mall RK, Agarwal A, Garg RK, Kar AM, Shukla R (2003). Short course of prednisolone in Indian patients with solitary cysticercus granuloma and new-onset seizures. Epilepsia 44(11):1397-1401.

69. Prakash S, Garg RK, Kar AM, et al. (2006). Intravenous methyl prednisolone in patients with solitary cysticercus granuloma: a random evaluation. Seizure 15(5):328-332.

70. Singla M, Prabhakar S, Modi M, Medhi B, Khandelwal N, Lal V (2011). Short-course of prednisolone in solitary cysticercus granuloma: A randomized, doubleblind,placebo-controlled trial. Epilepsia 52(10): 1914-1917.

71. Thussu A, Arora A, Prabhakar S, Lal V, Sawhney IM (2002). Acute symptomatic seizures due to single CT lesions: how long to treat with antiepileptic drugs? Neurology India 50(2):141-144.

72. Gupta M, Agarwal P, Khwaja GA, et al.(2002). Randomized prospective study of outcome of short term antiepileptic treatment in small single enhancing CT lesion in brain. Neurology India 50(2):145-147.
73. Verma A, Misra S (2006). Outcome of short-term antiepileptic treatment in patients with solitary cerebral cysticercus granuloma. Acta Neurol Scand 113(3): 174-177.

74. Rajshekhar V, Jeyaseelan L (2004). Seizure outcome in patients with a solitary cerebral cysticercus granuloma. Neurology 62(12): 2236-2240.

75. Nash TE, Del Brutto OH, Butman JA, et al. (2004). Calcific neurocysticercosis and epileptogenesis. Neurology 62(11): 1934-1938.

76. Pradhan S, Kathuria MK, Gupta RK (2000). Perilesional gliosis and seizure outcome: a study based on magnetization transfer magnetic resonance imaging in patients with neurocysticercosis. Ann Neurol 48(2):181-187.

77. Kaushal S, Rani A, Chopra SC, Singh G (2006). Safety and efficacy of clobazam versus phenytoinsodium in the antiepileptic drug treatment of solitary cysticercus granulomas. Neurology India 54(2):157-160. 